\title{
Reputation with Noisy Precommitment ${ }^{1}$
}

\author{
David K. Levine \\ Cesar Martinelli ${ }^{2}$
}

December 5, 1995

Revised August 13, 1997

\begin{abstract}
We investigate a game in which there is a long-run player who has private information about how long will the game last, who must precommit to an action, and who faces a sequence of short-run players who get a noisy signal of that action. We might expect such a long-lived long-run player to behave as a Stackelberg leader since most short-run players will eventually learn the true action. However, short-run players know that a long-run player that has been in the game for a long time must have chosen the Stackelberg action and may ignore the evidence from the signal. If so, the long-run player may have no reason to actually choose the Stackelberg action. We show that if there is a chance that the long-run player chooses other action by mistake, and the signal is sufficiently informative, this paradox can be resolved.
\end{abstract}

\footnotetext{
${ }^{1}$ We thank Michele Boldrin, Marco Celentani, and especially an Associate Editor for helpful comments and suggestions. Levine gratefully acknowledges support from the Spanish Ministry of Education DGICYT Grant SA95-0159, the UCLA Academic Senate, and National Science Foundation Grant SBR-93-20695. Martinelli gratefully acknowledges support from the Spanish Ministry of Education DGICYT Grant DD94-0378.

${ }^{2}$ Levine: Departments of Economics, UCLA, CA 90024; Martinelli: Departamento de Economía, Universidad Carlos III de Madrid, 28903 Getafe, Spain. This research was conducted while Levine was visiting at Carlos III.
} 


\section{Introduction}

Consider a computer firm that is opening a store. The store will sell two types of computers, IBM's and Macintoshes. There are a fixed number of consumers that will purchase computers from the store. Due to good market research, the firm knows how many customers will wish to visit the store. Different customers will put the computers they buy to different uses; some will be better off with IBM's and some with Macintoshes. Consumers will arrive at the store one at a time, and each will explain to the salesperson what they wish to use the computer for. The salesperson will then determine whether the consumer should buy an IBM or a Macintosh. There are two types of sales people, and the firm must decide which type to employ. For a relatively low wage, it can hire an ignorant salesperson who will ignore what the consumer says, and assign an IBM or Macintosh at random; for a higher wage it can hire a knowledgeable salesperson who will assign the correct type of computer. Because of the overhead in hiring a salesperson, the firm will hire one person, and employ that person until demand is exhausted and the store closed.

Consumers must decide whether to buy one computer (for the office) or two (one for the office and one for the home). If they knew for sure that they would be assigned the correct type of computer, they would prefer to buy two computers; if they believe that they are being assigned a computer at random, they wish to buy only a computer for the office. Unlike the firm, the consumers do not know the size of the market, but they do share common probabilistic beliefs about how large it is. However, they observe a noisy signal of how satisfied previous customers of the store are.

The firm would rather pay extra for the knowledgeable salesperson if the consumer will buy two computers instead of one. 
If the market size is relatively small, the firm has little reason to employ the knowledgeable salesperson: consumers will acquire little information about the salesperson, and will decide whether to buy one or two computers based upon their prior beliefs about whether the salesperson is knowledgeable or not. The firm should simply save the extra wage. If the market size is relatively large, we would expect the firm to hire the knowledgeable salesperson since most of the arriving consumers will have learned from the experience of their predecessors whether the salesperson is most likely knowledgeable or not. This suggests that the equilibrium should be characterized by a critical market size: all smaller sizes hire the ignorant salesperson and all larger sizes hire the knowledgeable salesperson. This however leads to a paradox: the consumers know the strategy of the firm, and so if the firm has been in business long enough, the consumers will know that the firm must have hired the knowledgeable salesperson. Any evidence from the signal is disregarded, and attributed to noise. As a result, the long-lived firm has no reason to actually hire the knowledgeable salesperson: once it has been in business long enough consumers will believe that the knowledgeable salesperson was hired even if he was not. So the longlived firm does best by hiring the ignorant salesperson.

The goal of this paper is to show how this paradox can be resolved if two assumptions are satisfied. First, there must be the possibility of the firm making a mistake, that is hiring a person they think is knowledgeable who is in fact not. In this case no matter how long the firm has been in business consumers will never be certain the firm has successfully hired the knowledgeable salesperson, and will never 
disregard the signal of how satisfied previous customers are. ${ }^{4}$ Second, there are two different sources of information about the behavior of the firm: the signal and the length of time the firm has been in business. The paradox will be resolved if the signal is relatively more important.

Although we present our model in terms of a firm which must determine the quality level of a new product, the issues raised in this paper apply more generally. The relevant setting is one in which there is a long-run player who has private information about how long will the game last, who must precommit to some action, and who faces a sequence of short-run players who get a noisy signal of that action. In this setting, it is an equilibrium for the short-run players to ignore the signal and for the long-run player to play the low cost action irrespective of how long will the game last. When we introduce the probability of making mistakes ("mandatory types") this ceases to be an equilibrium, because the signal always reveals information about the presence of the mandatory types. Moreover, it becomes possible to have an equilibrium characterized by a critical market size (length of life of the long-run player), as the short-run players do not ignore the signal even after it becomes common knowledge that the market is larger than the critical size. ${ }^{5}$

In order to build some intuition for our results, note that the stage game played by the firm and the consumers has a prisoner's dilemma structure. In a one-shot situation, the only equilibrium would be for the firm to produce low quality and for

\footnotetext{
${ }^{4}$ A similar point is made in Allen and Faulhaber [1988]: they argue in a two-period model that in order for consumers to pay attention to past experience, it is necessary to have some noise in the decision process of the firm.

${ }^{5}$ We will show that when the size of the market is common knowledge from the beginning of the game, there must be a range over which the long-run player mixes: intuitively, if the long-run player is thought to be using the low cost action, the gain to using the high-cost action is larger than if he is thought to be using the high cost action. This is because the "low cost" signal is much less likely in the former case. Consequently, there is a range where whichever action the long-run player is using he
} 
the consumer to be mean. If the firm is allowed to precommit, however, it will precommit to the high quality in order to convince the consumer to be nice. This possibility is lost if the consumer only gets a noisy signal of the action played by the firm: in a pure strategy equilibrium, the consumer should ignore the value of the signal. But this distroys the incentive for the firm to produce high quality. In this sense, the paradox we identify is similar to the one described by Bagwell [1995]: in a two-period, two-player game where the action of the first player is communicated to the second player with noise, a pure equilibrium strategy of the sequential game is a Nash equilibrium of the simultaneous move game. In other words, with noise, the first mover advantage is lost. Our setup, however, is different from Bagwell's: we consider a dynamic game in which the noise goes to zero as time goes on. More importantly, our focus is not the paradox in itself but a proposed solution. ${ }^{6}$

Since the solution we propose relies on private information, we can relate our work to the literature on reputation and in particular to the model studied by Diamond [1989]. While in our model the length of the game does not depend on the action chosen by the long-run player, in Diamond's model the length of the game depends only on the behavior of the long-run player. (In the Diamond model, there is no precommitment, however.) Unlike the Diamond model, in our setting, reputation can increase or decrease over time, while in the Diamond setting, once a bad signal is received the game is over.

In a context in which the tenure of a firm is determined by how profitable it is in a particular market, the Diamond story is more applicable. Where the tenure of the firm is determined instead by the size of the market, as in the computer story above, or 
by other exogenous factors, such as the health of the proprietor, opportunities in other markets, and so forth, the model set forth here is more appropriate.

With the exception of Diamond's [1989] model and related models such as that of Martinelli [1996], traditional reputational models ${ }^{7}$ have focused on how the possibility of committed types allows uncommitted firms to reap the benefits of precommitment. Typically, in equilibrium, the long-run player initially plays the precommitment strategy, and late in the game falls back on the low cost strategy. In the early rounds, the short-run player therefore plays as a Stackelberg follower. This focus on the final rounds of play seems misplaced from a practical point of view. Here we eliminate cheating in the final rounds by forcing firms to precommit, and focus instead on the fact that in the early rounds short-lived firms will follow the low cost strategy. If the probability of this is viewed as sufficiently high by the consumers, they will not play as Stackelberg followers, and the long-lived firms must make a costly investment in reputation in order to enjoy its benefits later in the game. Such a description of play seems much closer to what happens in many markets, as firms must actually weigh the costs of building a reputation against its benefits.

\section{The Model}

We first describe the stage game. The long-run player, player 1, must choose an action $a_{1}$ of either high $\operatorname{cost} \bar{c}$ or low $\operatorname{cost} \underline{c}$ where $\bar{c}>\underline{c}$. The short-run player, player 2, must choose an action $a_{2}$ of either being nice $N$ or mean $M$. The utility of player 1 is $u_{1}\left(a_{2}\right)-a_{1}$, where $u_{1}(N)>u_{1}(M)$. More strongly, we assume that $u_{1}(N)-\bar{c}>u_{1}(M)-\underline{c}$, so that it is worth precommitting to the high cost action if

\footnotetext{
${ }^{6} \mathrm{We}$ leave for later a discussion of another solution proposed by van Damme and Hurkens [1994].
} 
doing so induces the short-run player to be nice rather than mean. It is useful to define the ratio between the cost difference between the long-run player's two actions, and the utility difference between the short-run players two actions as

$$
k_{1}=\frac{\bar{c}-\underline{c}}{u_{1}(N)-u_{1}(M)} .
$$

Under our assumptions $0<k_{1}<1$.

The preferences of the short-run player are characterized by a number $1>k_{2}>0$. If $\alpha_{1}$ is the probability that the long-run player uses the high cost action, then the short-run player will be willing to be nice if $\alpha_{1} /\left(1-\alpha_{1}\right) \geq k_{2}$ and is indifferent between nice and mean if this holds with exact equality. (He will wish to be mean if the inequality is reversed.)

The dynamic game takes place in continuous time. There is a continuum of short-run players who arrive sequentially, one at a time. The mass of short-run players, which is the same as the length of life of the long-run player, is denoted by $T \in(0, \infty)$. The long-run player knows this. The short-run players have a common knowledge prior over these times given by a cumulative distribution over the positive reals $F$ with corresponding continuous density $f$. We will always assume that $f$ is bounded above. If $f$ is unbounded at the origin, our results are unchanged but this case adds additional complications to the analysis.

Some of our results depend on restricting how rapidly the probability of $T$ declines as $T \rightarrow \infty$. Specifically, we say that the probability of $T$ declines slowly if $\sup f(t) /(1-F(t))<\infty$. It may easily be checked that this assumption is satisfied for relatively thick tailed distributions such as the Pareto and exponential, and fails for

\footnotetext{
${ }^{7}$ For example, Kreps and Wilson [1982], Milgrom and Roberts [1982] and Fudenberg and Levine
} 
thinner tailed distributions such as the truncated normal and the chi-squared.

Long-run players are infinitely patient. Our results, of course, still hold if they discount the future at a very small rate. They must choose a fixed value of $a_{1}$ to be applied to all short-run players. This may be contingent on $T$. Independently of $T$, long-run players may be one of three types. The first two types are referred to as mandatory types: the first type plays $\bar{c}$ no matter what; the second type plays $\underline{c}$ no matter what. The third type is called the optional type and may choose which strategy to play. The probability of the first two types are denoted by $\mu(\bar{c})$ and $\mu(\underline{c})$, respectively. A (pure) strategy $\sigma_{1}$ for the (optional) long-run player is simply a measurable map from $T$ to $\{\bar{c}, \underline{c}\} .^{8}$

Given the long-run players choice of $a_{1}$ a scalar signal $\eta_{t}$ observed by both players is generated. For example, this might be a cumulative measure of the favorable publicity the product has received over time. This signal follows a Wiener process with drift $m\left(a_{1}\right)$ and fixed instantaneous variance $v{ }^{9}$ The drift may be viewed as the rate at which publicity accumulates, while the random motion of the process reflects the fact that publicity is an imperfect measure of product quality. The assumption that the instantaneous variance $v$ is independent of the action simplifies computations.

According to this model the unconditional cumulative distribution of $\eta_{t}$ given $a_{1}$ is $\Phi\left(\left(\eta_{t}-t m\left(a_{1}\right)\right) / \sqrt{t v}\right)$ where $\Phi$ is the standard normal distribution. We will

[1989].

${ }^{8}$ With mandatory types, the restriction to pure strategies plays no role beyond the simplification of notation. Without mandatory types, we cannot disregard the possibility of mixed strategy equilibria (see Section 5).

${ }^{9}$ This is the continuous time limit of a discrete process $\eta_{t}=\eta_{t-1}+m\left(a_{1}\right) \Delta t+\varepsilon_{t}$, where $\Delta t$ is the length of a period, and $\varepsilon_{t}$ are (not necessarily normal) random variables with mean zero and variance $v \Delta t$. 
use $\phi$ to denote the standard normal density. For convenience we define $\bar{m}=m(\bar{c})$ and $\underline{m}=m(\underline{c})$. We assume (without loss of generality) that $\bar{m}>\underline{m}$. This corresponds to the convention that $\eta_{t}$ measures favorable (as opposed to unfavorable) publicity. ${ }^{10}$ A strategy profile for the short-run players is then a measurable map from $\left(t, \eta_{t}\right)$ to probability distributions over $\{N, M\}$.

The beliefs of the short-run player may be represented by the posterior odds ratio between the probability of the long-run player playing $\bar{c}$ to his probability of playing $\underline{c}$. We denote this by $\gamma_{t}$. Let $\bar{F}_{t}\left(\sigma_{1}\right)$ denote the fraction of the original population of player 1 's who are playing $\bar{c}$ at time $t$, and let $\underline{F}_{t}\left(\sigma_{1}\right)$ be the corresponding number playing $\underline{c}$. Then by Bayes law

$$
\gamma_{t}\left(\sigma_{1}, \eta_{t}\right)=\frac{\phi\left(\left(\eta_{t}-t \bar{m}\right) / \sqrt{t v}\right) \bar{F}_{t}\left(\sigma_{1}\right)}{\phi\left(\left(\eta_{t}-t \underline{m}\right) / \sqrt{t v}\right) \underline{F}_{t}\left(\sigma_{1}\right)}
$$

An equilibrium consists of a strategy of the long-run player $\hat{\sigma}_{1}(T)$ and of the short-run player $\hat{\sigma}_{2}\left(t, \eta_{t}\right)$ such that $\hat{\sigma}_{1}$ maximizes

$$
\int_{0}^{T} \int_{\eta_{t}}\left[u_{1}\left(\hat{\sigma}_{2}\left(t, \eta_{t}\right)\right)-\sigma_{1}(T)\right] \phi\left(\frac{\eta_{t}-t m\left(\sigma_{1}(T)\right)}{\sqrt{t v}}\right) d\left(\frac{\eta_{t}-t m\left(\sigma_{1}(T)\right)}{\sqrt{t v}}\right) d t
$$

and such that

$$
\hat{\sigma}_{2}\left(t, \eta_{t}\right)= \begin{cases}N & \gamma_{t}\left(\hat{\sigma}_{1}, \eta_{t}\right)>k_{2} \\ M & \gamma_{t}\left(\hat{\sigma}_{1}, \eta_{t}\right)<k_{2}\end{cases}
$$

The equilibrium thus defined is a perfect Bayesian equilibrium because at any stage of the game strategies are optimal given the beliefs, and the beliefs are obtained from equilibrium strategies and the observed signal using Bayes' rule.

\footnotetext{
${ }^{10}$ Note that we do not require $\underline{m}<0$; it may simply be that if the product is of low quality it receives
} 
We say that the strategy $\sigma_{1}$ has the cutoff property if there is a $\widetilde{T}$ such that $\sigma_{1}(T)=\underline{c}$ for $T<\tilde{T}$ and $\sigma_{1}(T)=\bar{c}$ for $T>\tilde{T}$. We say the equilibrium has the cutoff property if the player 1 strategy has the cutoff property.

\section{Existence of Cutoff Equilibria}

Our main results are

Theorem 1: Suppose that $f(t)>0$ for all $t>0{ }^{11}$ and that $\mu(\bar{c}), \mu(\underline{c})>0$. Then, there exists $\Delta$ such that if $(\bar{m}-\underline{m}) / \sqrt{v} \geq \Delta$ an equilibrium with the cutoff property exists.

Theorem 2: Suppose that $f(t)>0$ for all $t>0$ and that $\mu(\bar{c}), \mu(\underline{c})>0$. If, in addition, the probability of $T$ declines slowly, there exists $\Delta$ such that if $(\bar{m}-\underline{m}) / \sqrt{v} \geq \Delta$ all equilibria have the cutoff property.

The first theorem states that, if the signal is sufficiently informative about the action chosen by the long-run player, there is an equilibrium in which they will choose the low cost action if their time horizon is short and the high cost action if their time horizon is long. The second theorem gives us a sufficient condition for that to be the only type of equilibrium.

The idea of the proofs is that a long-run player who expect to be in the game long enough to reap the benefits of building a reputation will choose to do so by committing to the high cost action. The situation is complicated by the fact that, if there were no mandatory types, short-run players would become convinced at some 
point that they are facing a long-run player committed to the high cost action, thus disregarding completely the value of the signal. But that would destroy the reputational incentive to choose the high cost action in the first place. We avoid this paradox using $\mu(\underline{c})>0$. Then, short-run players can never be certain they are facing a long-run player committed to the high cost action, so they will never disregard completely the value of the signal. Moreover, if the probability of $T$ declines slowly, then the short-run players do not suddenly become "too confident" that the long-run player is playing the high cost leading them to disregard a bad realization of the value of the signal.

We proceed via several lemmas, proven in the Appendix. First we need some additional notation. Let $\eta_{t}\left(\sigma_{1}\right)$, given implicitly by $\gamma_{t}\left(\sigma_{1}, \eta_{t}\left(\sigma_{1}\right)\right)=k_{2}$, be the value of the signal that makes the short-run player at time $t$ indifferent between being nice and being mean if the long-run player is expected to play according to the strategy $\sigma_{1}$. Define $\underline{z}_{t}\left(\sigma_{1}\right)=\left(\eta_{t}\left(\sigma_{1}\right)-t \underline{m}\right) / \sqrt{t v}$ and $\bar{z}_{t}\left(\sigma_{1}\right)=\left(\eta_{t}\left(\sigma_{1}\right)-t \bar{m}\right) / \sqrt{t v}$. These represent cutoff values for the short-run player playing the nice action when the signal is normalized to be standard normal conditional on the long-run player actually playing the low cost $\left(\underline{z}_{t}\left(\sigma_{1}\right)\right)$ and high cost $\left(\bar{z}_{t}\left(\sigma_{1}\right)\right)$ actions. From these cutoffs, we may compute $\underline{p}_{t}\left(\sigma_{1}\right)=\int_{\underline{z}_{t}\left(\sigma_{1}\right)}^{\infty} \phi(z) d z$ to be the probability that $\gamma_{t}\left(\sigma_{1}, \eta_{t}\right)$ is greater or equal to $k_{2}$ (that is, that the nice action is chosen) conditional on the long-run player playing the low action, and $\bar{p}_{t}\left(\sigma_{1}\right)=\int_{\bar{z}_{t}\left(\sigma_{1}\right)}^{\infty} \phi(z) d z$ to be the corresponding number conditional on the high cost action.

\footnotetext{
${ }^{11}$ With some extra work, it can be shown that $f(t)>0$ need only hold for sufficiently small $t$ (the same applies to Theorem 2).
} 
To determine the strategy used by the long-run player, we will compute the gain to using the high cost strategy over the low cost strategy over the lifetime $T$. This is given by

$$
\int_{0}^{T} \int_{\eta_{t}}\left[u_{1}\left(\hat{\sigma}_{2}\left(t, \eta_{t}\right)\right)-\bar{c}\right] \frac{1}{\sqrt{t v}} \phi\left(\frac{\eta_{t}-t \bar{m}}{\sqrt{t v}}\right) d \eta_{t} d t-\int_{0}^{T} \int_{\eta_{t}}\left[u_{1}\left(\hat{\sigma}_{2}\left(t, \eta_{t}\right)\right)-\underline{c}\right] \frac{1}{\sqrt{t v}} \phi\left(\frac{\eta_{t}-t \underline{m}}{\sqrt{t v}}\right) d \eta_{t} d t .
$$

Given the equilibrium strategy of the short-run players and the definitions of $\bar{p}_{t}\left(\sigma_{1}\right)$ and $\underline{p}_{t}\left(\sigma_{1}\right)$, this is equivalent to

$$
\int_{0}^{T}\left(\bar{p}_{t}\left(\sigma_{1}\right)\left[u_{1}(N)-u_{1}(M)\right]+\left[u_{1}(M)-\bar{c}\right]\right) d t-\int_{0}^{T}\left(\underline{p}_{t}\left(\sigma_{1}\right)\left[u_{1}(N)-u_{1}(M)\right]+\left[u_{1}(M)-\underline{c}\right]\right) d t .
$$

We may divide through by $u_{1}(N)-u_{1}(M)$ and find a "normalized" gain from using the high cost action which simplifies to

$$
\bar{G}\left(\sigma_{1}, T\right)=\int_{0}^{T}\left(\bar{p}_{t}\left(\sigma_{1}\right)-\underline{p}_{t}\left(\sigma_{1}\right)-k_{1}\right) d t .
$$

In the special case where $\sigma_{1}$ is a cutoff strategy with cutoff $\widetilde{T}$ we abbreviate this to $\bar{G}(\widetilde{T}, T)$.

In equilibrium, therefore, a long-run player who lives $T$ must play the high cost strategy whenever $\bar{G}\left(\sigma_{1}, T\right)$ is strictly positive and the low cost strategy whenever it is strictly negative. Note that $\bar{G}\left(\sigma_{1}, T\right)$ is continuous in $T$. It follows that the longrun players will use a cutoff strategy if, as a function of $T, \bar{G}\left(\sigma_{1}, T\right)$ has a unique zero and is negative for sufficiently small values of $T$. The first Lemma gives conditions for this to be the case.

$\underline{\text { Lemma 1: }}$ If in equilibrium $\frac{d \bar{p}_{t}\left(\sigma_{1}\right)}{d t}>\frac{d \underline{p}_{t}\left(\sigma_{1}\right)}{d t}, \lim _{t \rightarrow 0}\left(\bar{p}_{t}\left(\sigma_{1}\right)-\underline{p}_{t}\left(\sigma_{1}\right)\right)=0$, and 
$\lim _{t \rightarrow \infty}\left(\bar{p}_{t}\left(\sigma_{1}\right)-\underline{p}_{t}\left(\sigma_{1}\right)\right)=1$, then the long-run player is using a strategy with the cutoff property.

The second Lemma shows that very early in the game the action of the shortrun player is unrelated to the action chosen by the long-run player. It also shows that late in the game the short-run player plays the nice action (with very high probability) if the long-run player has chosen the high cost action. This is the first of the sufficient conditions for the long-run player to use a cutoff strategy.

Lemma 2: If $\mu(\bar{c}), \mu(\underline{c})>0, \quad$ then $\quad \lim _{t \rightarrow 0}\left(\bar{p}_{t}\left(\sigma_{1}\right)-\underline{p}_{t}\left(\sigma_{1}\right)\right)=0 \quad$ and $\lim _{t \rightarrow \infty}\left(\bar{p}_{t}\left(\sigma_{1}\right)-\underline{p}_{t}\left(\sigma_{1}\right)\right)=1$ uniformly in the strategy $\sigma_{1}$ of the long-run player. If, in addition, $\sigma_{1}$ is a cutoff strategy with cutoff $\tilde{T}$ then $\bar{p}_{t}\left(\sigma_{1}\right), \underline{p}_{t}\left(\sigma_{1}\right)$ are continuous in $t, \tilde{T}$ for $t>0$.

Our third Lemma gives a sufficient condition for the probability of being nice to increase more rapidly at every moment in time if the high cost action is taken than if the low cost action is taken. This is the other sufficient condition needed to establish that the long-run player is using a cutoff strategy.

Lemma 3: Suppose that

$$
(\bar{m}-\underline{m}) / \sqrt{v}>2\left[\frac{1-\mu(\underline{c})-\mu(\bar{c})}{\min \{\mu(\underline{c})(1-\mu(\underline{c})), \mu(\bar{c})(1-\mu(\bar{c}))\}} \sup _{t \leq T}\left\{\frac{f(t)}{1-F(t)}\right\}\right]^{1 / 2} .
$$


Then for $t \leq T$ and any strategy $\sigma_{1}$ of the long-run player $\frac{d \bar{p}_{t}\left(\sigma_{1}\right)}{d t}>\frac{d \underline{p}_{t}\left(\sigma_{1}\right)}{d t}$. In addition, if for a particular $t \frac{d \underline{F}_{t}\left(\sigma_{1}\right) / d t}{\underline{F}_{t}\left(\sigma_{1}\right)}=\frac{d \bar{F}_{t}\left(\sigma_{1}\right) / d t}{\bar{F}_{t}\left(\sigma_{1}\right)}$ then $\frac{d \bar{p}_{t}\left(\sigma_{1}\right)}{d t}>\frac{d \underline{p}_{t}\left(\sigma_{1}\right)}{d t}$.

The hypothesis of Lemma 3 incorporates the idea that the probability of nice and mean types must be positive (since otherwise the denominator on the RHS is zero and the condition cannot hold). It measures the relative importance of two sources of information about the long-run player: the rate at which his action is revealed via the signal (the LHS) and the probability that the game continues to be played. The latter is represented by the ratio of the density of $T$ to the probability of a larger value of $T$. If this ratio is large, then it is unlikely the game will continue much longer, and the fact that it does continue is therefore very informative. If it is too informative relative to the signal, then the short-run players may conclude (if the long-run player is using a cutoff strategy, for example) that despite bad realizations of the signal the long-run player is likely to be playing the high cost action. This then leads to the paradox described in the introduction, since the long-run player then has no incentive to actually use the high cost action, as the short-run player will be nice anyway.

The final Lemma shows that increasing the informativeness of the signal increases the utility from the costly action.

$\underline{\text { Lemma 4}: ~ H o l d i n g ~ f i x e d ~} k_{1}, k_{2}$ and $F$, if $\left(\bar{m}^{\prime}-\underline{m^{\prime}}\right) / \sqrt{v^{\prime}}>(\bar{m}-\underline{m}) / \sqrt{v}$ then for any $\sigma_{1}, T \bar{G}^{\prime}\left(\sigma_{1}, T\right)>\bar{G}\left(\sigma_{1}, T\right)$.

We now prove the Theorems at the start of the section. 
Proof of Theorem 1: To show that a cutoff equilibrium actually exists, we must show that there is a solution to $\bar{G}(T, T)=0$ and that when the long-run player uses the corresponding cutoff strategy $\sigma_{1}, \frac{d \bar{p}_{t}\left(\sigma_{1}\right)}{d t}>\frac{d \underline{p}_{t}\left(\sigma_{1}\right)}{d t}$, so that we may apply Lemma 1.

If we can show that $\bar{G}(T, T)$ is negative for $T$ sufficiently small, positive for $T$ sufficiently large and continuous in between, existence of a solution to $\bar{G}(T, T)=0$ will follow from the intermediate value theorem.

Suppose, then, that the long-run player is using a cutoff strategy $\tilde{\sigma}_{1}$ with cutoff $\tilde{T}$. From Lemma 2 it follows that $\bar{p}_{t}\left(\tilde{\sigma}_{1}\right)-\underline{p}_{t}\left(\tilde{\sigma}_{1}\right)$ is continuous in $t, \tilde{T}$ for $t>0$, and that as $t \rightarrow \infty \bar{p}_{t}\left(\tilde{\sigma}_{1}\right)-\underline{p}_{t}\left(\tilde{\sigma}_{1}\right) \rightarrow 1$ and $\bar{p}_{t}\left(\tilde{\sigma}_{1}\right)-\underline{p}_{t}\left(\tilde{\sigma}_{1}\right) \rightarrow 0$ uniformly in $\tilde{T}$. Since $\bar{p}_{t}\left(\tilde{\sigma}_{1}\right)-\underline{p}_{t}\left(\tilde{\sigma}_{1}\right)$ is also bounded between -1 and 1 , we conclude that $\bar{G}(T, T)$ is continuous in $T$, and, since $0<k_{1}<1$, that for some $\varepsilon>0$ and all $0<t<\varepsilon$ $\bar{G}(t, t)<0$, and for some $\tilde{T}<\infty \bar{G}(\tilde{T}, \tilde{T})>0$. This implies that $\bar{G}(T, T)=0$ has a solution.

It remains to verify that $\frac{d \bar{p}_{t}\left(\sigma_{1}\right)}{d t}>\frac{d \underline{p}_{t}\left(\sigma_{1}\right)}{d t}$. In the case of a probability of $T$ that declines slowly, this follows immediately from Lemma 3. In the general case, we may let $\bar{T}$ be the largest (sup) value of $T$ such that the hypothesis of Lemma 3 holds. Obviously, as $(\bar{m}-\underline{m}) / \sqrt{v}$ goes to infinity, so does $\bar{T}$. On the other hand, from the argument above, for any value of $(\bar{m}-\underline{m}) / \sqrt{v}$ there is a largest $T$ solving $\bar{G}(T, T)=0$. By Lemma 4, we see that as $(\bar{m}-\underline{m}) / \sqrt{v}$ increases, $T$ cannot increase, 
and indeed, must actually decrease. It follows that for some $\Delta$ and all $(\bar{m}-\underline{m}) / \sqrt{v} \geq \Delta, \bar{T}>T$. By the conclusion of Lemma $3 \frac{d \bar{p}_{t}\left(\sigma_{1}\right)}{d t}>\frac{d \underline{p}_{t}\left(\sigma_{1}\right)}{d t}$ for $t \leq \bar{T}$. On the other hand, for $t>T$,

$$
\frac{d \underline{F}_{t}\left(\sigma_{1}\right) / d t}{\underline{F}_{t}\left(\sigma_{1}\right)}=\frac{d \bar{F}_{t}\left(\sigma_{1}\right) / d t}{\bar{F}_{t}\left(\sigma_{1}\right)}=\frac{f(t)}{1-F(t)}
$$

since only the mandatory low cost types are choosing the low cost action. Again by Lemma $3, \frac{d \bar{p}_{t}\left(\sigma_{1}\right)}{d t}>\frac{d \underline{p}_{t}\left(\sigma_{1}\right)}{d t}$, so this holds for all $t$ as desired.

Proof of Theorem 2: The fact that all equilibria have the cutoff property when the probability of $T$ declines slowly for all sufficiently large $(\bar{m}-\underline{m}) / \sqrt{v}$ follows from Lemma 3 which guarantees in this case that $\frac{d \bar{p}_{t}\left(\sigma_{1}\right)}{d t}>\frac{d \underline{p}_{t}\left(\sigma_{1}\right)}{d t}$. Combining this with Lemma 2 gives the hypothesis of Lemma 1 and the desired conclusion.

Theorem 1 does not preclude the existence of non-cutoff equilibria, that is, equilibria in which $\bar{G}\left(\hat{\sigma}_{1}(T), T\right)$ has several zeros. To make sure that the equilibrium strategy of the long-run player has the cutoff property we need the restriction on the tails of the distribution, incorporated in Theorem 2. 


\section{Comparative Statics}

We turn in this section to the comparative statics of the cutoff point. This is determined by the way in which $\bar{G}$ changes when the parameters are modified. The following Lemma follows immediately from the definition of $\bar{G}$ :

Lemma 5: Holding fixed $\bar{m}, \underline{m}, v, k_{2}$ and $F$, if $k_{1}^{\prime}<k_{1}$ then for any $\sigma_{1}, T$ $\bar{G}^{\prime}\left(\sigma_{1}, T\right)>\bar{G}\left(\sigma_{1}, T\right)$

Combining this with Lemma 4, we see that $\bar{G}$ is increasing in the informativeness of the signal and decreasing in the ratio between the gain from using the low cost action to the benefit from the short-run player being nice. The implications of this for comparative statics can be seen in Figure 1.

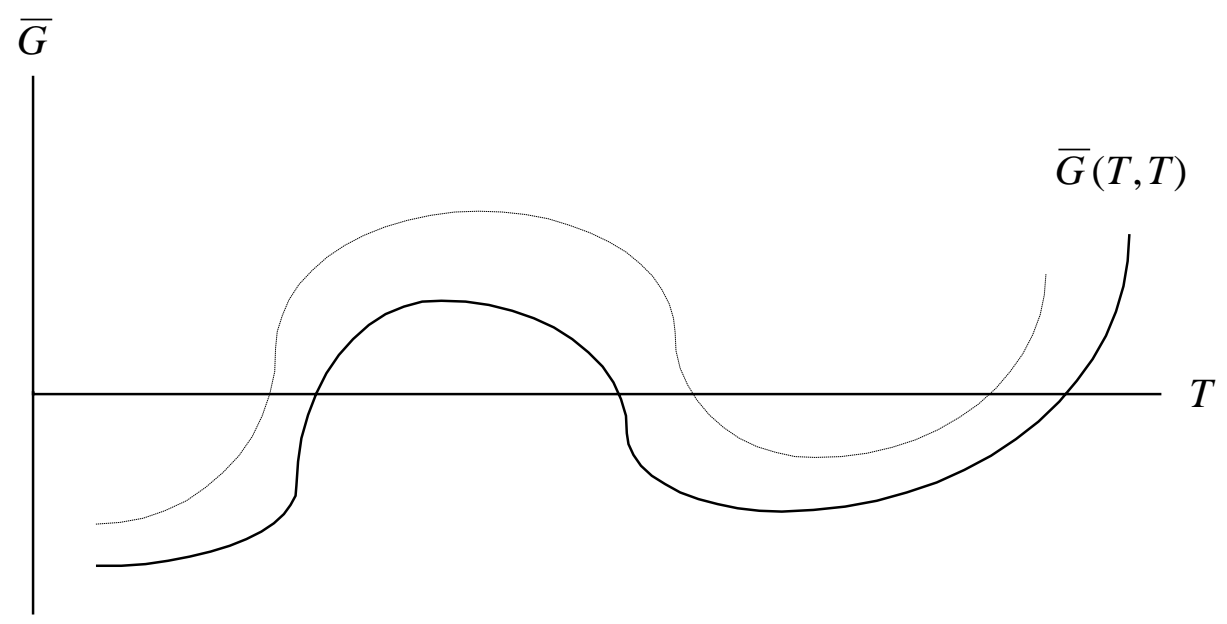

As $\bar{G}$ increases, both the best and worst possible equilibrium cutoff points shift to the right. Generally speaking, then, we see that the range of equilibria shifts toward shorter cutoffs as the informativeness of the signal increase or the ratio between the 
gain from using the low cost action to the benefit from the short-run player being nice decreases. An equilibrium cutoff point in the middle, however, could move in the opposite direction, thus yielding perverse results in terms of comparative statics.

\section{The Role of Private Information}

In our model there are two sources of private information. On the one hand short-run players do not know whether the long run player is a normal type or a mandatory type; on the other hand they do not know for how long will the long-run player will stay in the market. We discuss the two assumptions separately in this section.

Due to the paradox described in the introduction, the existence of mandatory types is crucial for the existence of cutoff equilibrium. Without mandatory types, any equilibrium in which the long-run player chooses the high cost action for some $T$ must involve either randomizing behavior or alternating intervals in $T$ of the high cost and low cost action. Moreover, we cannot disregard a pure strategy equilibrium in which the long-run player chooses the low cost action in spite of $T$, and all short-run players choose to be mean in spite of the signal.

It is instructive to compare our work with that of van Damme and Hurkens [1994]. They use a setup similar to Bagwell's [1995], that is, a two-person sequential decision model without mandatory types. They argue that the most plausible equilibrium is a mixed strategy equilibrium that generates an outcome close to the Stackelberg outcome and converges to the Stackelberg outcome as the noise level goes to zero. Van Damme and Hurkens use an equilibrium refinement that implies 
that, for small noise, players coordinate expectations on the equilibrium involving the least amount of randomization from player 1 needed to keep player 2 from disregarding the signal. We avoid the use of mixed strategies by introducing some exogenous probability of making mistakes, and give a condition under which all equilibria exhibit the cutoff property. We might add that the use of an equilibrium refinement that involves some sort of coordination of expectations is at least problematic in our setup, with a continuum of short-run players.

In the absence of private information about the length of stay of the long-run player in the market, the signal becomes the only source of information about the behavior of the firm. It might be tempting to think that, as the signal improves over time, there must be a cutoff equilibrium in the model thus modified. Lemma 6 gives us a sufficient condition for this not to be the case:

Lemma 6: In the modified game (with complete information about $T$ ), if $\mu(\underline{c}) \leq \mu(\bar{c})<1 / 2$, there is no cutoff equilibrium.

(The proof is in the Appendix). Lemma 6 holds regardless of how informative the signal is.

The idea of the proof is the following. Regardless of the action actually chosen by the long-run player, the gain from using the high cost action is negative for $T$ sufficiently small but positive for $T$ sufficiently large. However, the gain from using the high cost action is larger when the short-run players expect a normal longrun player to use the low cost rather than the high cost action. The reason for this is that when the low cost action is thought to be used by the normal type, it becomes easy to persuade the short-run players that they are facing the mandatory type playing 
the high cost. Hence, there is an interval $\left(T^{\prime}, T^{\prime \prime}\right)$ in which the gain from using the high cost action is positive if the short-run players expect a normal long-run player to use the low cost action but negative if they expect him to use the high cost action. For $T \in\left(T^{\prime}, T^{\prime \prime}\right)$, equilibrium behavior involves the long-run playerEMBED using a mixed strategy. ${ }^{12}$

With uncertain length of life, the effect described above is offset by the fact that non-mandatory types are dropping out over time. In terms of our original model, we can think of the short-run players as learning "too fast" if they know from the beginning of the game that $T>T^{\prime}$.

\section{Extensions}

It is interesting to ask whether the results in this paper can be extended to a more general class of games. Note that Lemmas 2 and 3 hold for any strategy of the long-run player (and hence, independently of any assumption about the payoffs to the long-run player). In other words, take any game of precommitment with the long-run, short-run player structure described in the paper, with a similar noise process, with mandatory types and private information about $T$. Let $\{\bar{c}, \underline{c}\}$ be the two possible actions of the long-run player. Then the difference between the probability that the short-run player who plays a best response at $t$ to $\bar{c}$ when the long-run player played $\bar{c}$ and that he plays a best response to $\bar{c}$ when the long-run player played $\underline{c}$ goes to zero when $t$ is close to zero and goes to one as $t$ goes to infinite. Moreover, under our fat-tails condition on beliefs about $T$, this difference is strictly increasing.

\footnotetext{
${ }^{12}$ If the condition stated in the Lemma is not met, there might be a cutoff equilibrium. Intuitively, when
} 
The implications of this result for the equilibrium strategy of the long-run player (Lemma 1) depend on the payoffs to the long-run player. Our results hold in any game with a prisoner's dilemma payoff structure, and in which the gain for the long-run player from adopting his one-shot equilibrium action is independent of the action of the short-run player. Even without private information about $T$, however, we can prove that any equilibrium strategy $\sigma_{1}$ for the long-run player satisfies the Stackelberg property: there is a $\widetilde{T}$ such that $\sigma_{1}(T)=\bar{c}$ for $T>\widetilde{T}$. This follows from the fact that $\bar{p}_{t}\left(\sigma_{1}\right)$ goes to one and $\underline{p}_{t}\left(\sigma_{1}\right)$ goes to zero as $t$ goes to infinite. Note that the Stackelberg property is weaker than the cutoff property.

We want to conclude by discussing an extension of our model to the issue of international direct investment. In our view, the most important decision made by sovereign countries with respect to international direct investment is the choice of institutions for the treatment of foreign investors. Such institutions include legal arrangements to discriminate against foreign investors in favor of domestic investors, legal restrictions on capital flows, and so forth.

To simplify matters, suppose there are only two sets of institutions for the treatment of foreign investors: "good institutions," corresponding to the high cost action in the model, and "bad institutions," corresponding to the low cost action in the model. A government has to choose the set of institutions having some private information about the expected length of time $T$ the institutions will be in place (say, until the next big political change, or until opportunities for foreign direct investment are exhausted). While investors do not get to observe directly the character of the 
institutions chosen by the government, they can observe the frequency of events such as restrictions on repatriation of funds, the difficulty in establishing joint ventures, legal obstacles to investment and so forth. To simplify matters, suppose there are only two types of investors: those with good alternative investment opportunities and those without them. The nice action of the short-run player corresponds to both types of investors investing; the mean action of the short-run player corresponds to only those with poor outside opportunities investing.

Under those circumstances, our model predicts that a country should suffer from a period of low investment before all foreign investors become convinced that it is worth to invest money in it. Moreover, this length of time varies randomly, depending on whether the country is lucky or unlucky with the random outcome of the signal.

This argument has important implications for the consequences of political stability and information flows on direct investment. In particular, it points out that it may well be necessary for a country to suffer through a period of low investment even if the country has set up a favorable institutional framework for the treatment of foreign investors. This focus is different than the usual discussion of reputational models of international lending, such as Eaton and Gersovitz [1981] and Bulow and Rogoff [1989]. In that discussion the issue addressed is whether reputation alone is sufficient to sustain foreign borrowing when the country has the option of defaulting in every period. These models do not suggest that there should be a delay in receiving funds from abroad, although Eaton [1990] does discuss the possibility that the interest rate paid may be higher initially than later on. Nor does any of the discussion indicate the possibility that the availability of funds may fluctuate randomly depending upon 
fortunate or unfortunate publicity. ${ }^{13}$ The point is that the model here provides an integrated account making relatively specific predictions of how information affects the rate of investment.

${ }^{13}$ Grossman and Van Huyck [1988] discuss the related issue of distinguishing justifiable from unjustifiable default. 


\section{References}

Allen, F. and G. Faulhaber [1988]: “Optimism Invites Deception,” Quarterly Journal of Economics, 103, 397-407.

Bagwell, K. [1995]: "Commitment and Observability in Games," Games and Economic Behavior, 8, 271-280.

Bulow, J. and K. Rogoff [1989]: "Sovereign Debt: Is to Forgive to Forget?" American Economic Review, 79, 43-50.

van Damme, E. and S. Hurkens [1994]: "Games with Imperfectly Observable Commitment," CentER discussion paper 9464. Games and Economic Behavior, forthcoming.

Diamond, D. [1989]: “Reputation Acquisition in Debt Markets," Journal of Political Economy, 97, 828-862.

Eaton, J. [1990]: "Sovereign Debt, Reputation and Credit Terms," NBER working paper 3424.

Eaton, J. and M. Gersovitz [1981]: "Debt with Potential Repudiation: Theory and Estimation," Review of Economic Studies, 48, 289-309.

Fudenberg, D. and D. Levine [1989]: "Reputation and Equilibrium Selection in Games with a Single Patient Player," Econometrica, 57, 759-778.

Grossman, H. and J. van Huyck [1988]: "Sovereign Debt as a Contingent Claim: Excusable Default, Repudiation, and Reputation," American Economic Review, 78, 1088-1097.

Kreps, D. and R. Wilson [1982]: "Reputation and Imperfect Information," Journal of Economic Theory, 27, 253-279.

Martinelli, C. [1996]: "Small Firms, Borrowing Constraints, and Reputation,” Journal of Economic Behavior and Organization, forthcoming.

Milgrom, P. and J. Roberts [1982]: "Predation, Reputation and Entry Deterrence," Journal of Economic Theory, 27, 280-312. 


\section{Appendix}

From the definitions of $\eta_{t}\left(\sigma_{1}\right)$ and $\gamma_{t}\left(\sigma_{t} \eta_{t}\right)$ we can compute an explicit expression for $\underline{z}_{t}\left(\sigma_{1}\right)$ and $\bar{z}_{t}\left(\sigma_{1}\right)$. Because we will continually make use of this expression, we summarize it as a Lemma.

\section{Lemma 0:}

$\underline{z}_{t}\left(\sigma_{1}\right)=\frac{\sqrt{t}(\bar{m}-\underline{m})}{2 \sqrt{v}}-\frac{\log \left(\frac{\bar{F}_{t}\left(\sigma_{1}\right)}{k_{2} \underline{F}_{t}\left(\sigma_{1}\right)}\right)}{\frac{\sqrt{t}}{\sqrt{v}}(\bar{m}-\underline{m})}, \bar{z}_{t}\left(\sigma_{1}\right)=-\frac{\sqrt{t}(\bar{m}-\underline{m})}{2 \sqrt{v}}-\frac{\log \left(\frac{\bar{F}_{t}\left(\sigma_{1}\right)}{k_{2} \underline{F}_{t}\left(\sigma_{1}\right)}\right)}{\frac{\sqrt{t}}{\sqrt{v}}(\bar{m}-\underline{m})}$.

We now prove the Lemmas in the text.

$\underline{\text { Lemma 1: }}$ : If in equilibrium $\frac{d \bar{p}_{t}\left(\sigma_{1}\right)}{d t}>\frac{d \underline{p}_{t}\left(\sigma_{1}\right)}{d t}, \lim _{t \rightarrow 0}\left(\bar{p}_{t}\left(\sigma_{1}\right)-\underline{p}_{t}\left(\sigma_{1}\right)\right)=0$, and $\lim _{t \rightarrow \infty}\left(\bar{p}_{t}\left(\sigma_{1}\right)-\underline{p}_{t}\left(\sigma_{1}\right)\right)=1$, then the long-run player is using a strategy with the cutoff property.

Proof: Since $\lim _{t \rightarrow 0}\left(\bar{p}_{t}\left(\sigma_{1}\right)-\underline{p}_{t}\left(\sigma_{1}\right)\right)=0$ for any strategy $\sigma_{1}$ of the long-run player and $k_{1}>0, \bar{G}\left(\sigma_{1}, T\right) \geq 0$ cannot hold for $T$ close to 0 . That is, there cannot be an equilibrium in which the long-run player chooses the high cost action for $T$ arbitrarily close to 0 .

Now, suppose there is a $\hat{T}$ such that $\bar{G}\left(\sigma_{1}, \hat{T}\right) \geq 0$, so that the long-run player may choose the high cost action for $T=\hat{T}$. Since $\lim _{t \rightarrow 0}\left(\bar{p}_{t}\left(\sigma_{1}\right)-\underline{p}_{t}\left(\sigma_{1}\right)\right)=0<k_{1}$, and, 
by the statement of the Lemma, $\bar{p}_{t}\left(\sigma_{1}\right)-\underline{p}_{t}\left(\sigma_{1}\right)$ is strictly increasing, it has to be the case that

$$
\bar{p}_{\hat{T}}\left(\sigma_{1}\right)-\underline{p}_{\hat{T}}\left(\sigma_{1}\right)>k_{1} .
$$

But then, since $\bar{p}_{t}\left(\sigma_{1}\right)-\underline{p}_{t}\left(\sigma_{1}\right)$ is strictly increasing, it has to be the case that

$$
\bar{p}_{t}\left(\sigma_{1}\right)-\underline{p}_{t}\left(\sigma_{1}\right)>k_{1} \text { for all } t>\hat{T},
$$

which implies $\bar{G}\left(\sigma_{1}, T\right)>0$ for all $T>\hat{T}$. That is, if in equilibrium the long-run player chooses the high cost action for some $T=\hat{T}$, he will choose it for any $T>\hat{T}$.

Finally, notice that, given that $k_{1}<1, \quad \lim _{t \rightarrow \infty}\left(\bar{p}_{t}\left(\sigma_{1}\right)-\underline{p}_{t}\left(\sigma_{1}\right)\right)=1$ and $\frac{d}{d t}\left(\bar{p}_{t}\left(\sigma_{1}\right)-\underline{p}_{t}\left(\sigma_{1}\right)\right)>0$, there must be some $\tilde{t}$ such that $\bar{p}_{t}\left(\sigma_{1}\right)-\underline{p}_{t}\left(\sigma_{1}\right)-k_{1}>0$ for all $t>\tilde{t}$. Notice also that this expression is strictly increasing, so that $\bar{G}\left(\sigma_{1}, T\right)-\bar{G}\left(\sigma_{1}, \tilde{t}\right)$ grows unboundedly with $T$. Since $\bar{G}\left(\sigma_{1}, \tilde{t}\right)$ is bounded from below by $-\left(1+k_{1}\right) \tilde{t}$, it cannot be true that $\bar{G}\left(\sigma_{1}, T\right) \leq 0$ for $T$ arbitrarily large. That is, there cannot be an equilibrium in which the long-run player chooses the low cost action for $T$ arbitrarily large.

Lemma 2: If $\quad \mu(\bar{c}), \mu(\underline{c})>0, \quad$ then $\quad \lim _{t \rightarrow 0}\left(\bar{p}_{t}\left(\sigma_{1}\right)-\underline{p}_{t}\left(\sigma_{1}\right)\right)=0 \quad$ and $\lim _{t \rightarrow \infty}\left(\bar{p}_{t}\left(\sigma_{1}\right)-\underline{p}_{t}\left(\sigma_{1}\right)\right)=1$ uniformly in the strategy $\sigma_{1}$ of the long-run player. If, in addition, $\sigma_{1}$ is a cutoff strategy with cutoff $\tilde{T}$ then $\bar{p}_{t}\left(\sigma_{1}\right), \underline{p}_{t}\left(\sigma_{1}\right)$ are continuous in $t, \tilde{T}$ for $t>0$. 
Proof: The first result, $\lim _{t \rightarrow 0}\left(\bar{p}_{t}\left(\sigma_{1}\right)-\underline{p}_{t}\left(\sigma_{1}\right)\right)=0$, follows directly from the fact that $\bar{p}_{t}\left(\sigma_{1}\right)-\underline{p}_{t}\left(\sigma_{1}\right)=\int_{\bar{z}_{t}\left(\sigma_{1}\right)}^{\underline{z}_{t}\left(\sigma_{1}\right)} \phi(z) d z$ and (from Lemma 0) $\underline{z}_{t}\left(\sigma_{1}\right)=\bar{z}_{t}\left(\sigma_{1}\right)+\frac{\sqrt{t}}{\sqrt{v}}(\bar{m}-\underline{m})$, and indeed, does not require the hypothesis that $\mu(\bar{c}), \mu(\underline{c})>0$. Notice that the rate of convergence can be bounded independently of $\sigma_{1}$, so that the convergence is uniform as required.

For the second result, $\lim _{t \rightarrow \infty}\left(\bar{p}_{t}\left(\sigma_{1}\right)-\underline{p}_{t}\left(\sigma_{1}\right)\right)=1$, we begin with $\underline{z}_{t}\left(\sigma_{1}\right)$ from Lemma 0 . Since the term $\log \left(\frac{\bar{F}_{t}\left(\sigma_{1}\right)}{k_{2} \underline{F}_{t}\left(\sigma_{1}\right)}\right)$ is bounded from above by $\log \left(\frac{1-\mu(\underline{c})}{k_{2} \mu(\underline{c})}\right)$ (independent of $\left.\sigma_{1}\right), \lim _{t \rightarrow \infty} \underline{z}_{t}\left(\sigma_{1}\right)=\infty$. It follows that $\lim _{t \rightarrow \infty} \underline{p}_{t}\left(\sigma_{1}\right)=0$.

Similarly, since the term $\log \left(\frac{\bar{F}_{t}\left(\sigma_{1}\right)}{k_{2} \underline{F}_{t}\left(\sigma_{1}\right)}\right)$ is bounded from below by $\log \left(\frac{\mu(\bar{c})}{k_{2}(1-\mu(\bar{c}))}\right), \lim _{t \rightarrow \infty} \bar{z}_{t}\left(\sigma_{1}\right)=-\infty$. It follows that $\lim _{t \rightarrow \infty} \bar{p}_{t}\left(\sigma_{1}\right)=1$. In both cases the rate of convergence is bounded independently of $\sigma_{1}$, so that convergence is uniform as required.

Finally, turning to the case of a cutoff strategy $\tilde{\sigma}_{1}$ by player 1

$$
\begin{aligned}
& \underline{F}_{t}\left(\tilde{\sigma}_{1}\right)=\left\{\begin{array}{cc}
\mu(\underline{c})(1-F(t))+(1-\mu(\underline{c})-\mu(\bar{c}))(F(\tilde{T})-F(t)) & t<\tilde{T} \\
\mu(\underline{c})(1-F(t)) & t>\tilde{T}
\end{array}\right. \\
& \bar{F}_{t}\left(\tilde{\sigma}_{1}\right)=\left\{\begin{array}{cc}
\mu(\bar{c})(1-F(t))+(1-\mu(\underline{c})-\mu(\bar{c}))(1-F(\tilde{T})) & t<\tilde{T} \\
(1-\mu(\underline{c}))(1-F(t)) & t>\tilde{T} .
\end{array}\right.
\end{aligned}
$$

Both $\underline{F}_{t}\left(\tilde{\sigma}_{1}\right), \bar{F}_{t}\left(\tilde{\sigma}_{1}\right)$ are continuous in $t, \tilde{T}$. From the definitions it follows that 
$\underline{z}_{t}\left(\tilde{\sigma}_{1}\right), \bar{z}_{t}\left(\tilde{\sigma}_{1}\right)$ are both continuous in $t, \tilde{T}$ for $t>0$, and that $\bar{p}_{t}\left(\tilde{\sigma}_{1}\right)-\underline{p}_{t}\left(\tilde{\sigma}_{1}\right)$ is continuous in $t, \tilde{T}$ for $t>0$.

च

Lemma 3: Suppose that

$$
(\bar{m}-\underline{m}) / \sqrt{v}>2\left[\frac{1-\mu(\underline{c})-\mu(\bar{c})}{\min \{\mu(\underline{c})(1-\mu(\underline{c})), \mu(\bar{c})(1-\mu(\bar{c}))\}} \sup _{t \leq T}\left\{\frac{f(t)}{1-F(t)}\right\}\right]^{1 / 2} .
$$

Then for $t \leq T$ and any strategy $\sigma_{1}$ of the long-run player $\frac{d \bar{p}_{t}\left(\sigma_{1}\right)}{d t}>\frac{d \underline{p}_{t}\left(\sigma_{1}\right)}{d t}$. In addition, if for a particular $t \frac{d \underline{F}_{t}\left(\sigma_{1}\right) / d t}{\underline{F}_{t}\left(\sigma_{1}\right)}=\frac{d \bar{F}_{t}\left(\sigma_{1}\right) / d t}{\bar{F}_{t}\left(\sigma_{1}\right)}$ then $\frac{d \bar{p}_{t}\left(\sigma_{1}\right)}{d t}>\frac{d \underline{p}_{t}\left(\sigma_{1}\right)}{d t}$.

Proof: We may differentiate $\underline{z}_{t}\left(\sigma_{1}\right)$ from Lemma 0 to find,

$$
\frac{d \underline{z}_{t}\left(\sigma_{1}\right)}{d t}=\frac{\bar{m}-\underline{m}}{4 \sqrt{t v}}+\frac{1}{2} \frac{\log \left(\frac{\bar{F}_{t}\left(\sigma_{1}\right)}{k_{2} \underline{F}_{t}\left(\sigma_{1}\right)}\right)}{t^{3 / 2} v^{-1 / 2}(\bar{m}-\underline{m})}-\frac{\frac{d \log \bar{F}_{t}\left(\sigma_{1}\right)}{d t}-\frac{d \log \underline{F}_{t}\left(\sigma_{1}\right)}{d t}}{\frac{\sqrt{t}}{\sqrt{v}}(\bar{m}-\underline{m})} .
$$

For $\underline{p}_{t}\left(\sigma_{1}\right)$ and $\bar{p}_{t}\left(\sigma_{1}\right)$ we compute

$$
\begin{aligned}
& d \underline{p}_{t}\left(\sigma_{1}\right) / d t=-\phi\left(\underline{z}_{t}\left(\sigma_{1}\right)\right) d \underline{z}_{t}\left(\sigma_{1}\right) / d t \\
& d \bar{p}_{t}\left(\sigma_{1}\right) / d t=-\phi\left(\bar{z}_{t}\left(\sigma_{1}\right)\right) d \bar{z}_{t}\left(\sigma_{1}\right) / d t .
\end{aligned}
$$

From this and various definitions, we compute 


$$
\begin{aligned}
\left(\frac{d \bar{p}_{t}\left(\sigma_{1}\right)}{d t}-\frac{d \underline{p}_{t}\left(\sigma_{1}\right)}{d t}\right) / \phi\left(\bar{z}_{t}\left(\sigma_{1}\right)\right)= & \frac{1}{2}\left(\frac{\bar{F}_{t}\left(\sigma_{1}\right)}{k_{2} \underline{F}_{t}\left(\sigma_{1}\right)}-1\right) \frac{\log \left(\frac{\bar{F}_{t}\left(\sigma_{1}\right)}{k_{2} \underline{F}_{t}\left(\sigma_{1}\right)}\right)}{t^{3 / 2} v^{-1 / 2}(\bar{m}-\underline{m})} \\
& +\left(\frac{\bar{m}-\underline{m}}{4 \sqrt{t v}}-\frac{\left.\frac{d \log \underline{F}_{t}\left(\sigma_{1}\right)}{d t}-\frac{d \log \bar{F}_{t}\left(\sigma_{1}\right)}{d t}\right)}{\frac{\sqrt{t}}{\sqrt{v}}(\bar{m}-\underline{m})}\right) \\
& +\left(\frac{\bar{F}_{t}\left(\sigma_{1}\right)}{k_{2} \underline{F}_{t}\left(\sigma_{1}\right)}\right)\left(\frac{\bar{m}-\underline{m}}{4 \sqrt{t v}}+\frac{\left.\frac{d \log \underline{F}_{t}\left(\sigma_{1}\right)}{d t}-\frac{d \log \bar{F}_{t}\left(\sigma_{1}\right)}{d t}\right)}{\frac{\sqrt{t}}{\sqrt{v}}(\bar{m}-\underline{m})}\right)
\end{aligned}
$$

Notice that, since $\phi\left(\bar{z}_{t}\left(\sigma_{1}\right)\right)>0$, it will be the case that $\frac{d \bar{p}_{t}\left(\sigma_{1}\right)}{d t}>\frac{d \underline{p}_{t}\left(\sigma_{1}\right)}{d t}$ as long as the RHS of the previous expression is positive. The first term in the RHS is always positive because $\left(\frac{\bar{F}_{t}\left(\sigma_{1}\right)}{k_{2} \underline{F}_{t}\left(\sigma_{1}\right)}-1\right)$ and $\log \left(\frac{\bar{F}_{t}\left(\sigma_{1}\right)}{k_{2} \underline{F}_{t}\left(\sigma_{1}\right)}\right)$ have the same sign.

The second and the third term will both be positive if

$$
\frac{\bar{m}-\underline{m}}{4 \sqrt{t v}}>\left|\frac{\frac{d \log \underline{F}_{t}\left(\sigma_{1}\right)}{d t}-\frac{d \log \bar{F}_{t}\left(\sigma_{1}\right)}{d t} \mid}{\frac{\sqrt{t}}{\sqrt{v}}(\bar{m}-\underline{m})}\right|
$$

or, equivalently,

$$
(\bar{m}-\underline{m}) / \sqrt{v}>2 \mid\left[\frac{d \underline{F}_{t}\left(\sigma_{1}\right) / d t}{\underline{F}_{t}\left(\sigma_{1}\right)}-\frac{d \bar{F}_{t}\left(\sigma_{1}\right) / d t}{\bar{F}_{t}\left(\sigma_{1}\right)}\right]^{1 / 2} .
$$

It follows directly that if $\frac{d \underline{F}_{t}\left(\sigma_{1}\right) / d t}{\underline{F}_{t}\left(\sigma_{1}\right)}=\frac{d \bar{F}_{t}\left(\sigma_{1}\right) / d t}{\bar{F}_{t}\left(\sigma_{1}\right)}$ then $\frac{d \bar{p}_{t}\left(\sigma_{1}\right)}{d t}>\frac{d \underline{p}_{t}\left(\sigma_{1}\right)}{d t}$.

For the case $t \leq T$, let

$$
\alpha_{T}\left(\sigma_{1}\right)= \begin{cases}0 & \sigma_{1}(T)=\underline{c} \\ 1 & \sigma_{1}(T)=\end{cases}
$$

By definition, 


$$
\begin{aligned}
& \underline{F}_{t}\left(\sigma_{1}\right)=\mu(\underline{c}) \int_{t}^{\infty} d F(T)+(1-\mu(\underline{c})-\mu(\bar{c})) \int_{t}^{\infty}\left(1-\alpha_{T}\left(\sigma_{1}\right)\right) d F(T) \\
& \bar{F}_{t}\left(\sigma_{1}\right)=\mu(\bar{c}) \int_{t}^{\infty} d F(T)+(1-\mu(\underline{c})-\mu(\bar{c})) \int_{t}^{\infty} \alpha_{T}\left(\sigma_{1}\right) d F(T) .
\end{aligned}
$$

We can rewrite the term in brackets in the RHS of $\left(^{*}\right)$ as

$$
\begin{aligned}
{\left[\frac{d \underline{F}_{t}\left(\sigma_{1}\right) / d t}{\underline{F}_{t}\left(\sigma_{1}\right)}-\frac{d \bar{F}_{t}\left(\sigma_{1}\right) / d t}{\bar{F}_{t}\left(\sigma_{1}\right)}\right] } & =\frac{\mu(\underline{c}) f(t)+(1-\mu(\underline{c})-\mu(\bar{c}))\left(1-\alpha_{t}\left(\sigma_{1}\right)\right) f(t)}{\mu(\underline{c}) \int_{t}^{\infty} d F(T)+(1-\mu(\underline{c})-\mu(\bar{c})) \int_{t}^{\infty}\left(1-\alpha_{T}\left(\sigma_{1}\right)\right) d F(T)} \\
& -\frac{\mu(\bar{c}) f(t)+(1-\mu(\underline{c})-\mu(\bar{c})) \alpha_{t}\left(\sigma_{1}\right) f(t)}{\mu(\bar{c}) \int_{t}^{\infty} d F(T)+(1-\mu(\underline{c})-\mu(\bar{c})) \int_{t}^{\infty} \alpha_{T}\left(\sigma_{1}\right) d F(T)} .
\end{aligned}
$$

Manipulating this expression and making use of the fact that $0 \leq \alpha_{T}\left(\sigma_{1}\right) \leq 1$ we find that the first condition in the Lemma is sufficient for the conclusion.

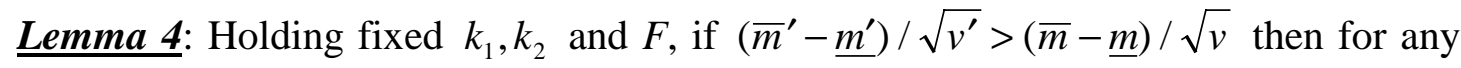
$\sigma_{1}, T \bar{G}^{\prime}\left(\sigma_{1}, T\right)>\bar{G}\left(\sigma_{1}, T\right)$

Proof: From the definition of $\bar{G}$ it suffices to show that

$$
{\overline{p^{\prime}}}_{t}\left(\sigma_{1}\right)-\underline{p}_{t}^{\prime}\left(\sigma_{1}\right)>\bar{p}_{t}\left(\sigma_{1}\right)-\underline{p}_{t}\left(\sigma_{1}\right) .
$$

Recall that $\bar{p}_{t}\left(\sigma_{1}\right)-\underline{p}_{t}\left(\sigma_{1}\right)$ is simply the integral of a standard normal density between $\bar{z}\left(\sigma_{1}\right)$ and $\underline{z}\left(\sigma_{1}\right)$. Moreover, $\bar{z}\left(\sigma_{1}\right)=-a+b, \underline{z}\left(\sigma_{1}\right)=a+b$, where $a$ is positive, the sign of $b$ is indeterminate, $a$ is increasing in $(\bar{m}-\underline{m}) / \sqrt{v}$ and $|b|$ is decreasing in $(\bar{m}-\underline{m}) / \sqrt{v}$. Since both increasing $a$ and decreasing $|b|$ increase the integral of any symmetric single peaked density over the range $[-a+b, a+b]$ the result follows. 
Lemma 6: In the modified game (complete information about T), if $\mu(\underline{c}) \leq \mu(\bar{c})<1 / 2$, there is no cutoff equilibrium.

Proof: As in Section 3, we can find a "normalized" gain for the long-run player from using the high cost action when $\sigma_{1}(T)=\bar{c}$ and a "normalized" gain for the long-run player from using the high cost action when $\sigma_{1}(T)=\underline{c}$. These are given, respectively, by

$$
\begin{aligned}
& \bar{G}(\bar{c}, T)=\int_{0}^{T}\left(\bar{p}_{t}(\bar{c})-\underline{p}_{t}(\bar{c})-k_{1}\right) d t, \\
& \bar{G}(\underline{c}, T)=\int_{0}^{T}\left(\bar{p}_{t}(\underline{c})-\underline{p}_{t}(\underline{c})-k_{1}\right) d t,
\end{aligned}
$$

with

$$
\begin{aligned}
& \bar{p}_{t}(\bar{c})=\int_{\bar{z}_{t}(\bar{c})}^{\infty} \phi(z) d z, \quad \underline{p}_{t}(\bar{c})=\int_{\underline{z}_{t}(\bar{c})}^{\infty} \phi(z) d z, \\
& \bar{p}_{t}(\underline{c})=\int_{\bar{z}_{t}(\underline{c})}^{\infty} \phi(z) d z, \quad \underline{p}_{t}(\underline{c})=\int_{\underline{z}_{t}(\underline{c})}^{\infty} \phi(z) d z,
\end{aligned}
$$

and

$$
\begin{aligned}
& \bar{z}_{t}(\bar{c})=-\frac{\sqrt{t}(\bar{m}-\underline{m})}{2 \sqrt{v}}-\frac{\log \left(\frac{1-\mu(\underline{c})}{k_{2} \mu(\underline{c})}\right)}{\frac{\sqrt{t}}{\sqrt{v}}(\bar{m}-\underline{m})}, \underline{z}_{t}(\bar{c})=\frac{\sqrt{t}(\bar{m}-\underline{m})}{2 \sqrt{v}}-\frac{\log \left(\frac{1-\mu(\underline{c})}{k_{2} \mu(\underline{c})}\right)}{\frac{\sqrt{t}}{\sqrt{v}}(\bar{m}-\underline{m})}, \\
& \bar{z}_{t}(\underline{c})=-\frac{\sqrt{t}(\bar{m}-\underline{m})}{2 \sqrt{v}}-\frac{\log \left(\frac{\mu(\bar{c})}{k_{2}(1-\mu(\bar{c}))}\right)}{\frac{\sqrt{t}}{\sqrt{v}}(\bar{m}-\underline{m})}, \underline{z}_{t}(\underline{c})=\frac{\sqrt{t}(\bar{m}-\underline{m})}{2 \sqrt{v}}-\frac{\log \left(\frac{\mu(\bar{c})}{k_{2}(1-\mu(\bar{c}))}\right)}{\frac{\sqrt{t}}{\sqrt{v}}(\bar{m}-\underline{m})} .
\end{aligned}
$$

Following the reasoning in Lemma 2, we can establish that $\bar{G}(\bar{c}, T), \bar{G}(\underline{c}, T)$ are continuous in $T$ and are negative for small enough $T$ and positive for large enough $T$. We want to prove that, under the conditions of the Lemma, $\bar{G}(\bar{c}, T)<0<\bar{G}(\underline{c}, T)$ for 
$T \in\left(T^{\prime}, T^{\prime \prime}\right)$ for some $T^{\prime}, T^{\prime \prime}$ so that for $T \in\left(T^{\prime}, T^{\prime \prime}\right)$ there is no equilibrium in pure strategies. Note that $\bar{G}(\bar{c}, T)<\bar{G}(\underline{c}, T)$ for all $T$ (and, in fact, $\bar{G}(\underline{c}, T)-\bar{G}(\bar{c}, T)$ is increasing in $T$ ) if

$$
\left|\log \left(\frac{\mu(\bar{c})}{k_{2}(1-\mu(\bar{c}))}\right)\right|<\left|\log \left(\frac{1-\mu(\underline{c})}{k_{2} \mu(\underline{c})}\right)\right|,
$$

which holds under the conditions in the Lemma (recall that $0<k_{2}<1$ ). This gives the desired result. 